\title{
Taxonomía y morfología de CRaticula gadorensis SP. NOV. (BACILLARIOPHYTA, StAuRONEIDACEAE)
}

\author{
TAXONOMY AND MORPHOLOGY OF CRATICULA GADORENSIS SP. NOV. \\ (BACILlariophyta, Stauroneidaceae)
}

\section{Saúl Blanco ${ }^{1, *}\left(\mathbb{D}\right.$, Adriana Olenici ${ }^{2} \mathbb{D}$, Fernando Ortega ${ }^{3,4} \mathbb{D}$, Francisco Jiménez- Gómez $^{3,4}$ (D) y Francisco Guerrero ${ }^{3,4}$ (D)}

1. Departamento de Biodiversidad y Gestión Ambiental, Facultad de Ciencias Biológicas y Ambientales, Universidad de León, Campus de Vegazana s/n, 24071, León, España. Laboratorio de diatomología y calidad de aguas. Instituto de Investigación de Medio Ambiente, Recursos Naturales y Biodiversidad. La Serna 58, 24007, León, España.

2. Babes-Bolyai University, Faculty of Environmental Science and Engineering, Cluj-Napoca, Rumanía.

3. Departamento de Biología Animal, Biología Vegetal y Ecología. Campus de Las Lagunillas, $\mathrm{s} / \mathrm{n}$. 23071 Jaén, España.

4. Centro de Estudios Avanzados en Ciencias de la Tierra. Campus de Las Lagunillas, s/n. 23071 Jaén, España.

*sblal@unileon.es

\section{Citar este artículo}

BLANCO, S., A. OLENICI, F. ORTEGA, F. JIMÉNEZ-GÓMEZ \& F. GUERRERO. 2019. Taxonomía y morfología de Craticula gadorensis sp. nov. (Bacillariophyta, Stauroneidaceae). Bol. Soc. Argent. Bot. 54: 5-11.

DOI: http://dx.doi. org/10.31055/1851.2372.v54. $\mathrm{n} 1.23574$

Recibido: 27 Agosto 2018 Aceptado: 2 Noviembre 2018 Publicado: 28 Marzo 2019 Editora: Eugenia Alicia Sar

\section{SUMMARY}

Background and Aims: The diatom Craticula gadorensis sp. nov. is described from samples of epilithic algae collected in a shallow pond from the Gador mountains, in the province of Almería (Spain).

M\&M: Samples were taken and processed following the usual protocols for diatoms. Morphological and ultrastructural characteristics of this new taxon are described and illustrated using light and scanning electron microscopy.

Results: The new species is characterised by having elliptic-lanceolate to rhombiclanceolate valves, with slightly protracted, subrostrate apices, a linear, narrow axial area and a slightly dilated, linear-lanceolate central area.

Conclusions: A morphological comparison with related species shows that the combination of features in Craticula gadorensis is unique (mainly the dimensions, areola density and the shape of the central area).

\section{KeY WORDS}

Diatoms, new species, shallow lakes, Almería, Spain.

\section{RESUMEN}

Introducción y objetivos: Se describe la diatomea Craticula gadorensis sp. nov. a partir de muestras de algas epilíticas recogidas en una balsa ganadera de la Sierra de Gádor, en la provincia de Almería.

M\&M: Las muestras se tomaron y procesaron siguiendo los protocolos metodológicos para diatomeas. Los caracteres morfológicos y ultraestructurales de este nuevo taxón se describen e ilustran mediante imágenes de microscopía óptica y electrónica.

Resultados: La nueva especie se caracteriza por presentar valvas elípticolanceoladas a rómbico-lanceoladas, con ápices ligeramente prolongados, subrostrados, con un área axial lineal, estrecha y un área central un poco dilatada, lineal-lanceolada.

Conclusiones: La comparación morfológica con otras especies del género muestra que la combinación de caracteres de Craticula gadorensis (principalmente las dimensiones, la densidad de areolas y la forma del área central) es única.

\section{Palabras clave}

Diatomeas, nueva especie, balsas ganaderas, Almería, España.

\section{INTRODUCCIÓN}

El género Craticula Grunow aparece en la literatura científica en el siglo XIX (Grunow, 1867) para designar un conjunto de especies de diatomeas capaces de generar "craticulae" o valvas internas. El género, con $C$. perrotettii Grunow como lectotipo designado por Robert Ross (Farr et al., 1979), fue considerado inválido durante mucho tiempo, al suponerse que estaba basado meramente en células aberrantes (Fourtanier \& Kociolek, 1999). Durante el siglo $X X$, las especies del género (principalmente $C$. cuspidata (Kütz.) D.G.Mann y afines) formaron la sección Orthostichae Cleve, y posteriormente los subgéneros Cuspidata (Grunow) Patrick 
y Halophila Patrick, dentro de Navicula Bory. Round et al. (1990) reestablecieron Craticula como género independiente, describiendo sus principales apomorfías. Finalmente, Morales \& Le (2005) lo hacen sinónimo de Lacunicula Lange-Bertalot, Cavacini, Tagliaventi \& Alfinito (2003) al transferir la especie tipo del género L. sardiniensis Lange-Bert., Cavacini, Tagliaventi \& Alfinito a Craticula. En su revisión del género, Mann \& Stickle (1991) muestran que Craticula no guarda una vinculación directa con las naviculáceas, asignando tentativamente el género a la familia Stauroneidaceae, criterio que se ha seguido hasta la fecha (Cox, 2015).

Los caracteres definitorios del género son: (i) la capacidad de formar "craticulae" y "estadios heribaudii" en las formas de resistencia como respuesta al estrés osmótico (no observados en todas las especies); (ii) un sternum elevado externa e internamente; (iii) estrías uniseriadas, generalmente paralelas, que continúan en un manto proporcionalmente estrecho, con areolas pequeñas y redondeadas ocluidas internamente por hímenes perforados; (iv) areolas externamente alineadas en estrías longitudinales rodeadas por costillas longitudinales, y (v) ausencia de costillas paralelas al sistema del rafe (Round et al., 1990; LangeBertalot, 2001). Además de ello, Craticula muestra diferencias evidentes con el género Navicula en el modo de división de los cloroplastos, en la estructura de los pirenoides y del cíngulo, y en varios aspectos de la reproducción sexual (Mann \& Stickle, 1991). El género comprende algo más de 50 especies (Beauger et al., 2017), una docena de las cuales habían sido citadas en la Península Ibérica a principios de siglo (Aboal et al., 2003), si bien durante los últimos años se han añadido nuevas especies. Entre las contribuciones recientes al género a nivel mundial destacan C. strelnikoviana E.Morales, S. Rivera \& C.E.Wetzel, C. guaykuruorum C.E.Wetzel, E.Morales \& Ector (in Morales et al., 2014) y $C$. lecohui Beauger, C.E.Wetzel \& Ector (in Beauger et al., 2017). Este artículo describe una nueva especie de Craticula hallada en la Balsa de la Chanata (Almería, España).

\section{Material y Métodos}

Las muestras fueron recogidas en primavera de 2017 en la zona litoral de Balsa de la Chanata
(Almería, España, 36 54'51.03”N $2^{\circ}$ 41'29.69”O, 1425 m s.n.m., Fig. 1), una balsa ganadera temporal situada en la Sierra de Gádor. Presenta una cuenca de recepción de algo más de 6 ha y una cobertura vegetal de la cuenca del 50\% (Frot et al., 2008). Las muestras fueron recogidas del epiliton de piedras litorales, con la ayuda de un cepillo y preservadas usando formaldehido $(4 \% \mathrm{v} / \mathrm{v})$. En el laboratorio se obtuvieron suspensiones de frústulos limpios mediante la oxidación de la materia orgánica con peróxido de hidrógeno $(30 \% \mathrm{v} / \mathrm{v})$, calentando a $70-90{ }^{\circ} \mathrm{C}$ para acelerar la reacción. Se añadieron, además, unas gotas de ácido clorhídrico $(3 \mathrm{M})$ para eliminar las inclusiones de carbonato cálcico. El montaje de las preparaciones microscópicas fue realizado usando una resina sintética de alto índice

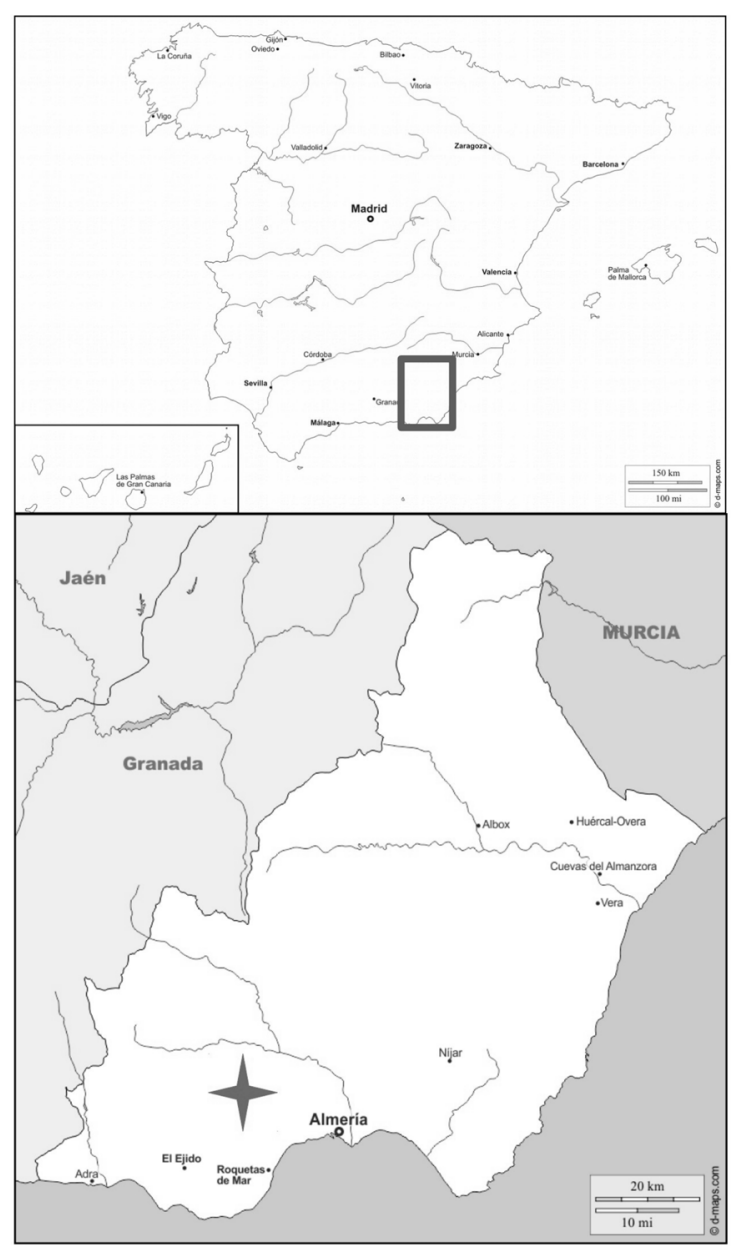

Fig. 1. Localización del sitio de muestreo. 
de refracción óptica (Naphrax $\left.{ }^{\circledR}\right)$. La identificación de las especies de diatomeas se realizó a 1000 aumentos, usando un microscopio óptico (MO) Olympus BX60, equipado con una cámara fotográfica OPTIKA, con la que fueron realizadas las fotografías de microscopía óptica. Las muestras fueron analizadas con microscopía electrónica de barrido (MEB) en la Unidad de Microscopía Electrónica de la Universidad de Jaén, colocando una gota de la muestra sobre una estructura metálica conductora y dejando secar a temperatura ambiente. Las muestras fueron posteriormente recubiertas con una capa de oro de $10 \mathrm{~nm}$ de grosor mediante un sistema de metalización modular de alto vacío (QUORUM Q150T ES). El estudio se realizó usando un microscopio MERLIN (Carl Zeiss), operando a $20 \mathrm{kV}$.

La relación entre los principales parámetros morfométricos en la población analizada fue estudiada representando las medidas realizadas en el espécimen tipo mediante un diagrama de dispersión.

\section{Resultados}

Craticula gadorensis S.Blanco sp. nov.

Valvae elliptico-lanceolatae ad rhombicolanceolatae apicibus leviter protractis subrostratis (Fig. 2). Longitudo 64-91 $\mu \mathrm{m}(81,5 \pm 10,3 \mu \mathrm{m})$, latitudo $16-20 \mu \mathrm{m}(18,2 \pm 1,1 \mu \mathrm{m})$, ratio $3,8-4,9$ $(4,5 \pm 0,4 \mu \mathrm{m})$. Area axialis linearis, angusta. Area centralis paullo dilatata in media aream axialem. Raphe recta filiformis. Striae transapicales parallelae, ad polos modice convergentes, 14-16 in $10 \mu \mathrm{m}(14,5 \pm 0,7$ in $10 \mu \mathrm{m})$. Areolae ca. 35 in 10 $\mu \mathrm{m} .11$ valvae mensae.

Holotypus. GDA-ALGAE 9107. Muestra de microscopía óptica y preparación de microscopía electrónica de barrido. Registro: http://phycobank. org/100518

Locus typicus. Balsa de la Chanata, Almería, España, sobre piedras $\left(36^{\circ} 54^{\prime} 51.03\right.$ ' $\mathrm{N}, 2^{\circ} 41^{\prime}$ 29.69” O, 1425 m s.n.m., 18-06-2017)

Etimología: "gadorensis", referido a la Sierra de Gádor, donde se ubica la localidad tipo.

Descripción. Valvas elíptico-lanceoladas a rómbico-lanceoladas, con ápices ligeramente prolongados, subrostrados (Fig. 2). Longitud: 64-91 $\mu \mathrm{m}(81,5 \pm 10,3 \mu \mathrm{m})$, anchura: $16-20 \mu \mathrm{m}(18,2$ $\pm 1,1 \mu \mathrm{m})$, cociente largo/ancho: 3,8-4,9 (4,5 \pm $0,4 \mu \mathrm{m})$ (Fig. 3). Área axial lineal, estrecha. Área central un poco dilatada, lineal-lanceolada. Rafe recto, filiforme. Estrías transapicales paralelas, algo convergentes hacia los polos, 14-16 en $10 \mu \mathrm{m}$ (14,5 $\pm 0,7$ en $10 \mu \mathrm{m})$. Medidas sobre 11 individuos.

Ultraestructura. Las valvas son planas y la transición hacia el estrecho manto es suave y gradual (Fig. 4D). El rafe yace sobre un sternum elevado tanto interna como externamente (Figs. 4A, B). Las fisuras proximales del rafe son ligeramente onduladas y ligeramente expandidas exteriormente (Fig. 4B), inconspicuas, ligeramente onduladas y puntiformes interiormente (Fig. 4C). El rafe termina apicalmente en la superficie valvar, en fisuras unciformes exteriormente (Fig. 4B), curvadas en el mismo sentido, e interiormente en fisuras ligeramente curvadas hacia el mismo lado de la valva, terminadas en helictoglosas simples,

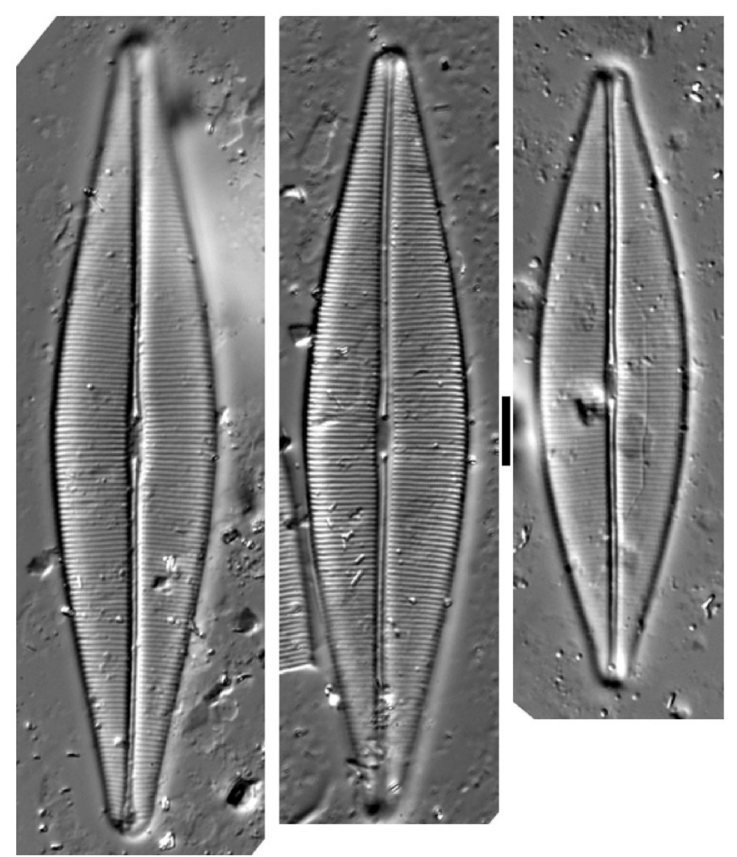

Fig. 2. Craticula gadorensis sp. nov. Individuos del especimen holotipo en vista valvar (MO, contraste interferencial). Barra de escala: $10 \mu \mathrm{m}$. 


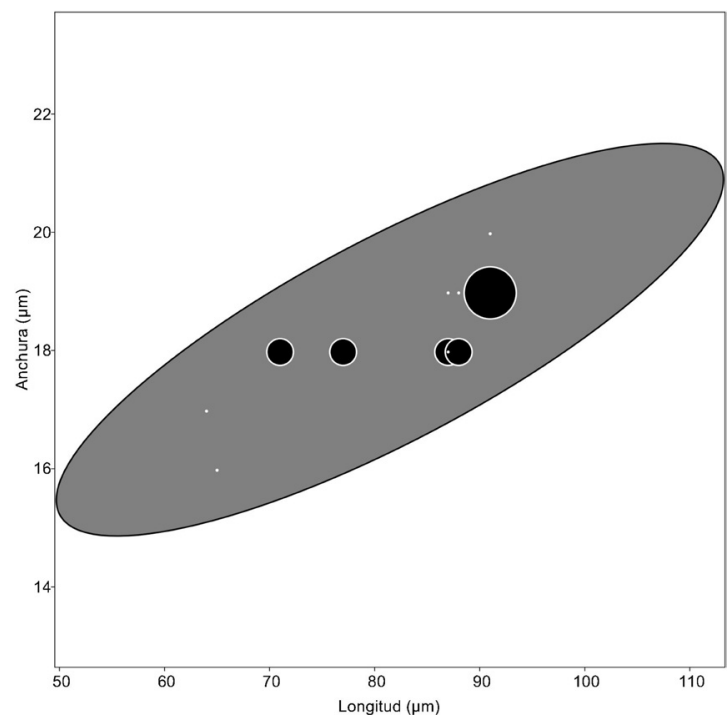

Fig. 3. Relación entre la longitud y la anchura de las valvas en la población tipo de Craticula gadorensis. El diámetro de los círculos es proporcional a la densidad de estrías. Puntos ajustados a una elipse de confidencia del $95 \%$. poco desarrolladas, que interrumpen la estriación, rodeando un área apical hialina (Fig. 4D). Las areolas son redondeadas internamente, apicalmente alargadas en vista externa (excepto las que rodean al rafe, que son puntiformes), dispuestas de forma regular y continua a lo largo de la estría ( $c a .35$ en 10 $\mu \mathrm{m})$, incluyendo el manto, y ocluidas internamente por hímenes (Fig. 4C). Se aprecian las costillas longitudinales en vista valvar externa (Fig. 4B).

Ecología. Las especies de Craticula son preferentemente bentónicas, en su mayoría típicas de aguas salobres, salinas o muy contaminadas y algunas especies son consideradas como las más tolerantes a la eutrofización (Levkov et al., 2016). Se ha observado una gran diversidad del género en hábitats acuáticos alcalinos y cálidos, incluyendo lagunas temporales y efímeras (Levkov et al., 2016). Las especies de este género presentan estrechos rangos de tolerancia a valores de $\mathrm{pH}$, conductividad y concentración de nutrientes (Beauger et al., 2017). En nuestro estudio, la población más importante de
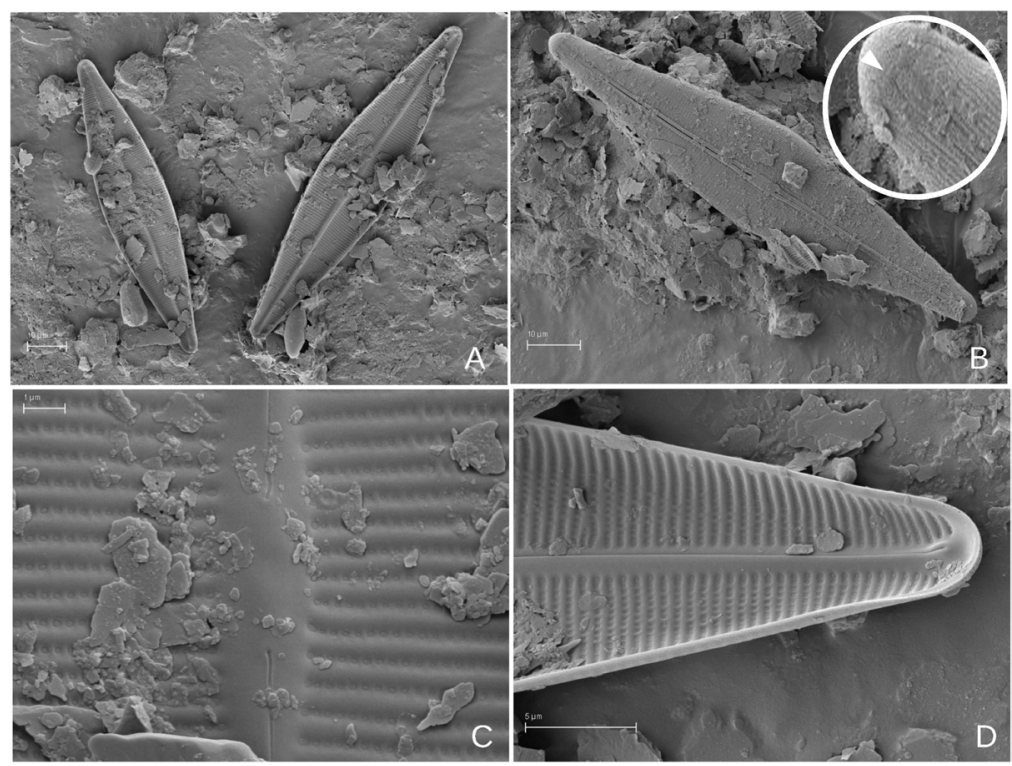

Fig. 4. Craticula gadorensis sp. nov. Individuos del especimen holotipo (MEB). A. Habitus. Vista valvar interna, obsérvese el sternum internamente elevado. B. Vista valvar externa. Obsérvese el detalle de un extremo distal del rafe, que termina en la cara valvar en una fisura unciforme (flecha), y las areolas insertas en costillas longitudinales. C. Detalle del nódulo central en vista interna. Obsérvese las terminaciones proximales del rafe, ligeramente onduladas, que concluyen en fisuras puntiformes, y las areolas redondeadas ocluidas por hímenes. D. Detalle del ápice valvar en vista interna. Obsérvese la suave transición entre el manto y la superficie valvar, y la pequeña helictoglosa que delimita un campo apical hialino. Escalas= A, B: $10 \mu \mathrm{m}$; C: $1 \mu \mathrm{m}$; D: $5 \mu \mathrm{m}$. 
C. gadorensis se encontró en la localidad tipo, donde constituye aproximadamente $1 / 3$ de la comunidad de diatomeas epilíticas. Este cuerpo de agua presentó bajos valores de conductividad $\left(0,29 \mathrm{mS} \mathrm{cm}^{-1}\right)$ y turbidez (131 NTU). La vegetación sumergida acompañante estuvo compuesta por las fanerógamas Ranunculus peltatus subsp. peltatus Moench y Zanichellia palustris L. y la carófita Tolypella prolifera (Ziz ex A.Braun) Leonh. El humedal mostró una cobertura de helófitos (Eleocharis palustris (L.) Roem. \& Schult.) del 70\% de la cubierta lagunar. Allí se encuentra acompañada de otras especies eurioicas y cosmopolitas como Achnanthidium minutissimum (Kütz.) Czarn., Encyonema lange-bertalotii Krammer o Gomphonema exilissimum (Grunow) Lange-Bert \& E.Reichardt que indican la presencia de aguas de baja conductividad y ligeramente alcalinas. También se hallaron algunas valvas de $C$. gadorensis en la cercana Balsa Blanca $\left(36^{\circ} 56^{\prime} 49.44\right.$ " $\mathrm{N}, 2^{\circ} 50^{\prime}$ 13.88" O, 1551 m s.n.m.), con similares especies botánicas (E. palustris, $R$. peltatus subsp. peltatus y $T$. prolifera, Ortega et al., 2004) y similares características limnológicas (conductividad $0,17 \mathrm{mS}$ $\mathrm{cm}^{-1}$; turbidez $52 \mathrm{NTU}$; alcalinidad $74 \mathrm{mg}^{-1}$ ).

\section{Discusión}

El conjunto de caracteres que presenta la población descrita es único dentro de Craticula y justifica la erección de una nueva especie, a saber: la combinación de un área central redondeada de unos $3 \mu \mathrm{m}$ de ancho, y la presencia de una densidad de areolas de $c a .35$ en $10 \mu \mathrm{m}$.
Diagnosis diferencial: las especies de Craticula pueden ser divididas en dos grandes grupos morfológicos: uno comprendido por taxones de gran tamaño en torno a C. cuspidata, capaces de desarrollar valvas internas, y otras especies más pequeñas, sin valvas internas conocidas, afines a C. subminuscula (Manguin) C.E.Wetzel \& Ector. Dadas sus dimensiones, Craticula gadorensis podría pertenecer al primer grupo, aunque no se han observado "craticulae" o "estadios heribaudii". La morfometría y morfología de C. gadorensis se solapa con siete especies (Tabla 1), todas ellas con valvas de más de $15 \mu \mathrm{m}$ de ancho. Las principales diferencias mostradas con estas especies son:

Craticula acidoclinata Lange-Bertalot \& Metzeltin (1996: 41, pl. 26, fig. 1-3) tiene un área central más ancha y areolas claramente visibles en $\mathrm{MO}$ (23-24 en $10 \mu \mathrm{m})$.

Craticula orientalis Metzeltin, Lange-Bertalot \& Nergi (2009: 23, pl. 71, figs 1-6) tiene valvas claramente rómbico-lanceoladas, con ápices no prolongados.

Craticula frenguellii Metzeltin, Lange-Bertalot \& García Rodríguez (2005: 36, pl. 96, figs 1-3, pl. 97, figs 1-6) se distingue claramente por presentar ápices notoriamente más ensanchados y redondeados, y una menor densidad de areolas por estría (ca. 24 en $10 \mu \mathrm{m}$, y no $c a .35$ como en C. gadorensis).

Craticula johnstoniae Bahls (2013: 22, figs 90-95) y C. sardiniana Bahls (2013: 23, figs 96-101) son dos especies similares descritas en Norteamérica. Ambas se distinguen fácilmente de C. gadorensis bajo el microscopio óptico por la forma elíptica

Tabla 1. Comparación morfométrica entre $C$. gadorensis sp. nov. y especies similares. '_': dato no publicado.

\begin{tabular}{|lcccc|}
\hline \multicolumn{1}{c}{ Especies } & Longitud $(\boldsymbol{\mu m})$ & Anchura $(\boldsymbol{\mu m})$ & Estrías en $\mathbf{1 0} \boldsymbol{\mu m}$ & Areolas en $\mathbf{1 0} \boldsymbol{\mu m}$ \\
\hline C. acidoclinata & $60-130$ & $16-24$ & $13-16$ & - \\
C. frenguellii & $42-110$ & $18-26$ & $14-15$ & 24 \\
C. johnstoniae & $78-105$ & $18-19$ & $14-17$ & 28 \\
C. obaesa & $59-90$ & $14-18$ & $13-15$ & 30 \\
C. orientalis & $85-125$ & $16-18$ & $17-18$ & $26-31$ \\
C. sardiniana & $68-113$ & $16-20$ & $14-18$ & $25-28$ \\
C. subpampeana & $80-110$ & $15-21$ & $13-17$ & 35 \\
C. gadorensis & $64-91$ & $16-20$ & $14-16$ & 30 \\
\hline
\end{tabular}


del área central, claramente diferenciada del área axial, lo que no ocurre en la especie aquí descrita. Además, los ápices valvares de $C$. johnstoniae son rostrados a subcapitados, y no subrostrados como en C. gadorensis. Según Bahls (2013), C. sardiniana sería conespecífica de la población identificada como "Craticula silviae nov. spec. prov.” en Lange-Bertalot 2001 (pl. 88, figs 1-5) que nunca se describió formalmente (pero véase Levkov et al., 2016). En cualquier caso, estos individuos hallados en charcas alcalinas efimeras de la isla de Cerdeña son proporcionalmente más alargados que $C$. gadorensis (relación largo/ancho: 4,0-6,5 vs. 3,8-4,9 según Seeligmann et al., 2008). Es de destacar que los ejemplares aquí descritos se parecen relativamente más a los ilustrados bajo el nombre de C. sardiniana en Levkov et al. (2016, figs 7: 1-3), que difícilmente se corresponden con esta especie según la descripción original.

Craticula subpampeana Van de Vijver \& Sterken (in Van de Vijver et al., 2010: 435, figs 37-45) difiere también en las proporciones de la valva (relación largo/ancho: 5,3) y en la presencia de un área central elíptica y no lineal. Obsérvese que, aunque el área central en Craticula se ha descrito invariablemente en la literatura como "estrecha" o "indistinta" (Mann \& Stickle, 1991; Beauger et al., 2017), tal descripción podría ser la excepción y no la regla para las especies de gran tamaño.

Craticula obaesa Van de Vijver, Kopalová \& Zidarova (2015: 38, figs 10-20) es, sin duda, la especie más parecida a $C$. gadorensis en términos de morfología y morfometría (Tabla 1). Fue descrita a partir de muestras de musgos recogidas en la Isla de James Ross, Mar de Weddell, por Van de Vijver et al. (2015). Sin embargo, la forma del área axial, lanceolada y expandiéndose gradualmente hacia el centro, donde forma un área central de unos 3 $\mu \mathrm{m}$ de ancho, permite diferenciarla fácilmente de C. gadorensis (compárense nuestra fig. 2 con las figs. 10-14 en Van de Vijver et al., 2015).

\section{Contribución de los autores}

FG y FO concibieron y diseñaron el estudio y realizaron el muestreo en campo, AO y FJG realizaron el estudio fotográfico y SB redactó el borrador del manuscrito.

\section{Agradecimientos}

Los autores quieren agradecer a la Junta de Andalucía los permisos concedidos para la toma de muestras en humedales de montaña de las Sierras Béticas de Andalucía. Este trabajo se enmarca en el proyecto "Humedales de montaña de Andalucia: inventario, tipologías y conservación" financiado por el Centro de Estudios Avanzados en Ciencias de la Tierra (CEACTierra) de la Universidad de Jaén. Agradecemos además las útiles correcciones de los revisores y editores del texto.

\section{Bibliografía}

ABOAL, M., M. ÁLVAREZ-COBELAS, J. CAMBRA \& L. ECTOR. 2003. Floristic list of non-marine diatoms (Bacillariophyceae) of Iberian Peninsula, Balearic Islands and Canary Islands. Updated taxonomy and bibliography. In: Witkowski, A. (ed.), Diatom Monographs 4. ARG Gantner, Rugell.

BAHLS, L. 2013. New diatoms (Bacillariophyta) from western North America. Phytotaxa 82: 7-28. http://dx.doi.org/10.11646/phytotaxa.82.1.2

BEAUGER, A., C. E. WETZEL, O. VOLDOIRE, A. GARREAU \& L. ECTOR. 2017. Morphology and ecology of Craticula lecohui sp. nov. (Bacillariophyceae) from hydrothermal springs (Puy-de-Dôme, Massif Central, France) and comparison with similar Craticula species. Nova Hedwigia Beih. 146: 7-22. http://dx.doi.org/10.1127/1438-9134/2017/007

COX, E. J. 2015. Coscinodiscophyceae, Mediophyceae, Fragilariophyceae, Bacillariophyceae (Diatoms). In: Frey, W. (ed.), Syllabus of Plant Families. Adolf Engler's Syllabus der Pflanzenfamilien, 13th ed., part 2/1, pp. 64-103. Borntraeger Science Publishers, Berlin.

FARR, E. R., J. A. LEUSSINK \& F. A. STAFLEAU. 1979. Index Nominum Genericorum (Plantarum). Regnum Veg. 100-102: 1-1896.

FOURTANIER, E. \& J.P. KOCIOLEK. 1999. Catalogue of the diatom genera. Diatom Res. 14: 1-190.

FROT, E., B. VAN WESEMAEL, A. S. BENET \& M. A. HOUSE. 2008. Water harvesting potential in function of hillslope characteristics: A case study from the Sierra de Gador (Almeria province, southeast Spain). J. Arid Environ. 72: 1213-1231. $\mathrm{http} / / /$ doi.org/10.1016/j.jaridenv.2007.12.009

GRUNOW, A. 1867. Reise seiner Majestät Fregatte Novara um die Erde. Botanischer Theil. 1. Algen. Aus der Kaiselich-Königlichen Hof-und Staasdruckerei, Botanischer Theil. 1. Algen. Wein. pp. 1-104. 
LANGE-BERTALOT, H. 2001. Navicula sensu stricto. 10 genera separated from Navicula sensu lato. Frustulia. In: Lange-Bertalot, H. (ed.), Diatoms Europe 2. A.R.G. Gantner, Ruggell.

LANGE-BERTALOT, H. \& D. METZELTIN. 1996. Indicators of oligotrophy - 800 taxa representative of three ecologically distinct lake types, Carbonate buffered - Oligodystrophic - Weakly buffered soft water. In: Lange-Bertalot, H. (ed.), Iconographia Diatomologica. Annotated Diatom Micrographs 2. Ecology, Diversity, Taxonomy. Koeltz Scientific Books, Koenigstein.

LANGE-BERTALOT, H., P. CAVACINI, N. TAGLIAVENTI \& S. ALFINITO. 2003. Diatoms of Sardinia. Rare and 76 new species in rock pools and other ephemeral waters. In: H. Lange-Bertalot (ed.), Iconographia Diatomologica. Annotated Diatom Micrographs 12. Biogeography-EcologyTaxonomy. Koeltz Scientific Books, Koenigstein.

LEVKOV,Z., S. TOFILOVSKA \& D. MITIC-KOPANJA. 2016. Species of the diatom genus Craticula Grunow (Bacillariophyceae) from Macedonia. Contrib. Sect. Nat. Math. Biotech. Sci. MASA 37: 129-165.

MANN, D. G. \& A. J. STICKLE. 1991. The genus Craticula. Diatom Res. 6: 79-107.

METZELTIN, D., H. LANGE-BERTALOT \& F. GARCÍA-RODRÍGUEZ. 2005. Diatoms of Uruguay. Compared with other taxa from South America and elsewhere. In: H. Lange-Bertalot (ed.), Iconographia Diatomologica. Annotated Diatom Micrographs 15. Taxonomy-BiogeographyDiversity. A.R.G. Gantner, Koenigstein.

METZELTIN, D., H. LANGE-BERTALOT \& S. NERGUI. 2009. Diatoms in Mongolia. In: H.
Lange-Bertalot (ed.), Iconographia Diatomologica. Annotated Diatom Micrographs 20. A.R.G. Gantner, Ruggell.

MORALES, E. A. \& M. LE. 2005. The taxonomy of the diatom Lacunicula sardiniensis Lange-Bertalot, Cavacini, Tagliaventi et Alfinito and its relationship with the genus Craticula Grunow (Bacillariophyceae). Proc. Acad. Nat. Sci. Phila. 154: 155-161.

MORALES, E. A., C. E. WETZEL, S. F. RIVERA, M. H. NOVAIS, L. HOFFMANN \& L. ECTOR. 2014. Craticula strelnikoviana sp. nov. and Craticula guaykuruorum sp. nov. (Bacillariophyta) from South American saline lakes. Nova Hedwigia Beih. 143: 223-237.

ORTEGA, F., M. PARACUELLOS \& F. GUERRERO 2004. Corología de macrófitos acuáticos en Andalucía oriental. Lazaroa 25: 179-185.

ROUND, F. E., R. M. CRAWFORD \& D. G. MANN. 1990. Diatoms: Biology and Morphology of the Genera. Cambridge University Press, Cambridge.

SEELIGMANN, C., N. I. MAIDANA \& M. MORALES. 2008. Diatomeas (Bacillariophyceae) de humedales de altura de la Provincia de Jujuy-Argentina. Bol. Soc. Argent. Bot. 43: 1-17.

VAN DE VIJVER, B., M. STERKEN, W. VYVERMAN, G. MATALONI, L. NEDBALOVA, K. KOPALOVA, J. ELSTER, E. VERLEYEN \& K. SABBE. 2010. Four new non-marine diatom taxa from the Subantarctic and Antarctic regions. Diatom Res. 25: 431-443. http://doi.org/10.1080/0269249X.2010.9705861

VAN DE VIJVER, B., K. KOPALOVÁ \& R. ZIDAROVA. 2015. Three new Craticula species (Bacillariophyta) from the Maritime Antarctic Region. Phytotaxa 213: 35-45. http://dx.doi.org/10.11646/phytotaxa.213.1.3 
